\title{
Asymptomatic Screening of Clients on Opioid Substitution Therapy for Tuberculosis: An Experience from India
}

\section{Banuru Muralidhara Prasad ${ }^{*}{ }^{(\mathbb{D},}$, Mandeep Singh2, Mukesh Gupta ${ }^{3}$, Pruthu Thekkur1, Rajeev Kumar ${ }^{4}$, Parmesh C. Bhatnagar ${ }^{4}$, Sarabjit Singh Chadha ${ }^{5}$}

\author{
${ }^{1}$ The International Union against Tuberculosis and Lung Disease (The Union), New Delhi, India \\ ${ }^{2}$ Medical Officer at OST Centre, Government of Punjab, Jalandhar, Punjab, India \\ ${ }^{3}$ Radiologist at Private Health Facility, Jalandhar, Punjab, India \\ ${ }^{4}$ Voluntary Health Association of India (VHAI), New Delhi, India \\ ${ }^{5}$ Foundation for Innovate New Diagnosis (FIND), New Delhi, India \\ Email: *bmprasad@theunion.org, *drprasadbm@gmail.com
}

How to cite this paper: Prasad, B.M., Singh, M., Gupta, M., Thekkur, P, Kumar, R., Bhatnagar, P.C. and Chadha, S.S. (2019) Asymptomatic Screening of Clients on Opioid Substitution Therapy for Tuberculosis: An Experience from India. Journal of Tuberculosis Research, 7, 131-134. https://doi.org/10.4236/jtr.2019.73012

Received: July 30, 2019

Accepted: September 1, 2019

Published: September 4, 2019

Copyright $\odot 2019$ by author(s) and Scientific Research Publishing Inc. This work is licensed under the Creative Commons Attribution International License (CC BY 4.0).

http://creativecommons.org/licenses/by/4.0/

\begin{abstract}
Clients on Opioid Substitution Therapy (OST) may be at risk of TB. We screened for tuberculosis in asymptomatic OST clients using Chest-X-ray (CXR) and sputum from those with CXR suggestive of TB were tested using Xpert MTB/Rif. Among 472 who underwent CXR, 1\% had CXR suggestive of TB. The number needed to screen was 118 clients for one CXR suggestive of TB. All four clients with CXR suggestive of TB underwent MTB/Rif assay and one was diagnosed with TB. The TB patient was asymptomatic and could have been missed through the passive approach. We recommend further studies to explore ACF among OST clients.
\end{abstract}

\section{Keywords}

OST, Tuberculosis, Active Case Finding, Screening, Chest X-Ray

Tuberculosis (TB) remains a major public health problem globally, especially in low-middle income countries (LMICs). World Health Organization (WHO) END TB has prioritized early diagnosis of TB through systematic and active screening among vulnerable groups as one of the key strategies to TB care and prevention [1]. National Strategic Plan 2017-25 (NSP) of India, in line with global strategy proposes to have focused interventions expanding the definitions of vulnerable populations to End-TB by 2025 [1]. Limited evidence suggests that clients on opioids may be considered as vulnerable population for developing 
tuberculosis [2].

Opioid substitution therapy (OST) programmes provide clients access to prescribe medicine such as methadone or buprenorphine which is usually administered orally under supervised clinical settings. OST clients are known to have high HIV prevalence, poor health-seeking behaviors, low nutritional status and high risk of TB [3]. A recent study from Ukraine showed a high number of TB detected among OST clients enrolled in programmes [4]. Few studies in the past have demonstrated high burden of Latent TB infection (LTBI) and Multi-Drug resistance TB among OST clients [3].

Considering these factors, clients on OST could be at higher risk of TB as many do not present with general TB symptoms like cough $\geq 2$ weeks, for the very nature of opioids [5]. As a result, client may remain undiagnosed and continue to spread TB infection that would jeopardise the mission to End TB.

We conducted a pilot Active Case Finding (ACF) for TB among OST clients attending a selected centre in the state of Punjab, India. The district TB programme officer, the chief medical officer at OST centre, and the district medical officer (civil surgeon) were briefed about the ACF activity. A private digital chest X-ray (CXR) with radiologist was engaged within $2 \mathrm{KMs}$ from the centre who offered CXR to all OST clients attending the centre during July 2016. Those clients willing to undergo CXR were accompanied by volunteers and CXR was provided free of cost to clients. Following the report of radiologist, CXR with abnormalities were read by district TB officer. The district TB officer reviewed the reports of clients with CXR abnormality suggestive of TB and offered Xpert MTB/Rif assay through the programme. The diagnosis of TB was made based on MTB/Rif assay results.

Figure 1 shows the process and outcomes of ACF of TB among asymptomatic OST clients. Of the total approached, $92.5 \%$ were willing to undergo CXR. This high proportion of willingness is appreciable and implies that it is feasible to conduct ACF among OST clients.

Among those who underwent CXR, 1\% had CXR suggestive of TB. The number needed to screen was 118 clients for one CXR suggestive of TB. All the four who had CXR suggestive of TB underwent MTB/Rif assay and one among them was found to be positive. The TB patient was asymptomatic and could have missed being diagnosed through passive approach. As these are preliminary results from a pilot study involving single centre with small sample size, it is difficult to comment on yield and utility of this ACF activity. Hence, we recommend for larger studies to explore the yield and utility of ACF among OST clients.

\section{Acknowledgements}

The team would like to acknowledge the support made by volunteers, the staff of OST centre, district TB officers, and authorities of district hospital. We would like to thank Mr Mohit who was the district co-ordinator from VHAI under Project Axshya. Authors would like to acknowledge the support from The Global Fund TB grant to India. 
OST clients attending clinic during study period and approached for screening for $\mathrm{TB}=510$

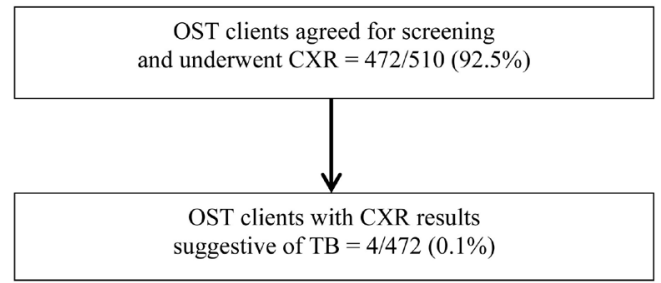

Xpert MTB/Rif examination of OST clients with CXR results suggestive of $\mathrm{TB}=4 / 4(100 \%)$

\section{Xpert MTB/Rif tested positive pulmonary TB among OST} clients with CXR results suggestive of $\mathrm{TB}=1 / 4(25 \%)$

One TB patient detected; completed the treatment

Figure 1. Schematic outline of screening OST client's for Tuberculosis.

\section{Funding}

Nil.

\section{Authors' Contributions}

$\mathrm{BMP}$ and $\mathrm{SC}$ conceptualized the project. $\mathrm{PB}, \mathrm{RK}, \mathrm{MS}$ and $\mathrm{MK}$ were involved in the implementation of the project in the selected district of Punjab. BMP, PB and RK were involved in data collection, analysis and feedback to implementation. PT was involved in the analysis of data and prepared the manuscript. All authors involved in critical review and approval of the final manuscript.

\section{Ethical Issues}

The study was embedded into the routine programme implementation with requisite permissions from department of health at the district level. Clients were verbally consented and referred by medical officer for Chest-X-ray at private facility.

\section{Conflicts of Interest}

The authors declare no conflicts of interest regarding the publication of this paper.

\section{References}

[1] Central TB Division (2017) National Strategic Plan for Tuberculosis Elimination 
2017-2025. New Delhi, India.

https://tbcindia.gov.in/WriteReadData/NSP\%20Draft\%2020.02.2017\%201.pdf

[2] Rao, R., Agarwal, A. and Ambekar, A. (2014) Opioid Substitution Therapy under National AIDS Control Programme: Clinical Practice Guidelines for Treatment with Buprenorphine. New Delhi, 50.

http://naco.gov.in/sites/default/files/Opiod\%20Substitution\%20Therapy\%20Guideli ne.pdf

[3] Deiss, R.G., Rodwell, T.C. and Garfein, R.S. (2009) Tuberculosis and Illicit Drug Use: Review and Update. Clinical Infectious Diseases, 48, 72-82. https://doi.org/10.1086/594126

[4] Islam, Z. (2014) Opioid Substitution Therapy and TB/MDR-TB: Example of Daily Dot, Experience of Integration and Motivation. In: 45th World Lung Conference, Barcelona, Spain. Barcelona, Spain: International Union against Tuberculosis and Lung Disease.

[5] Slawek, D.E., Lu, T.Y., Hayes, B. and Fox, A.D. (2019) Caring for Patients with Opioid Use Disorder: What Clinicians Should Know about Comorbid Medical Conditions. Psychiatric Research and Clinical Practice, 1, 16-26.

https://doi.org/10.1176/appi.prcp.20180005 